\title{
Effects of preexposure and retention interval placement on latent inhibition and perceptual learning in a choice-maze discrimination task
}

\author{
L. G. DE LA CASA \\ University of Seville, Seville, Spain \\ and \\ WILLIAM TIMBERLAKE \\ Indiana University, Bloomington, Indiana
}

\begin{abstract}
In two experiments, we examined how preexposure to discriminative stimuli and introduction of a 21-day retention interval affected the latent inhibition (LI) and perceptual learning (PL) of rats in a choice-maze discrimination task. Experimental groups were preexposed to three wall patterns, one in each of three arms of a maze. Control groups were preexposed only to white arms. PL groups were trained to discriminate A versus B, and LI groups, to discriminate A or B versus $\mathrm{C}$. The A and B patterns shared many elements not shared with the $\mathrm{C}$ pattern. In Experiment 1, both at the end of training and after the subsequent retention interval, the PL groups performed better than controls, whereas the LI groups performed worse. In Experiment 2, inserting the 21-day retention interval between preexposure and discrimination training disrupted final measures of LI but not PL performance. Implications for current concepts of PL and LI are discussed.
\end{abstract}

Latent inhibition (LI) occurs following preexposure to a neutral stimulus (i.e., a stimulus without a specific consequence) prior to its pairing with a consequence (for reviews, see Lubow, 1989; Lubow \& Gewirtz, 1995; Schmajuk, 2002). LI is measured in terms of an experimental group's delay in establishing an association between the preexposed neutral stimulus and a consequence, in comparison with a control group not preexposed to the neutral stimulus. From a functional point of view, LI is presumed to be a part of the adaptive process by which organisms learn to attend to stimuli likely to act as effective signals of consequences while they ignore other cues. Thus, limited attentional resources can be captured by novel and/or predictive stimuli rather than by more familiar ones.

However, LI is not the only consequence of stimulus preexposure. When two neutral complex stimuli are presented without consequences, an enhancement of their discriminability can occur (for reviews, see Hall, 1991; McLaren \& Mackintosh, 2000). This phenomenon has been labeled the perceptual learning (PL) effect, defined as "an increase in the ability to extract information from the environment, as a result of experience and practice with stimulation coming from it" (Gibson, 1969, p. 3).

This research was supported by Spanish Ministry of Education and Culture Grants PR2004-0256 and SEJ2004-00065 to the first author. The authors thank Oscar Pineño and Victoria D. Chamizo for their helpful comments on an earlier version of this article, and Cara Wellman for lending the maze. Correspondence concerning this article should be addressed to L. G. De la Casa, Department of Experimental Psychology, C/Camilo Jose Cela, s/n 41018 Sevilla, Spain (e-mail: delacasa@us.es).
Considering the similarities of the procedures that produce LI and PL effects, these two phenomena seem likely to have common processes. In fact, McLaren, Kaye, and Mackintosh (1989; see also McLaren \& Mackintosh, 2000) hypothesized that LI is an important underlying mechanism of the PL effect. They argued that a stimulus is composed of a set of elements, some shared with other stimuli and some unique to it. Because of the large number of shared elements in two similar stimuli, these elements should result in more total LI during preexposure. The resultant loss of attention and associability for these shared elements should enhance attention to and learning about unique elements of the stimuli during subsequent discrimination training. In contrast, because of the large number of unique elements in two differing stimuli, preexposure should produce LI to the majority of elements of both stimuli, thus retarding subsequent discrimination training between them.

In short, when an organism is confronted with a difficult discrimination between two stimuli (because they share many elements), preexposure to the stimuli should facilitate learning relative to a control with no preexposure (the PL effect). But when an organism is confronted with an easy discrimination (because the cues share few elements), preexposure should result in slower discrimination learning relative to a control with no preexposure (the LI effect). These predictions have been supported by research on discrimination of maze arms, which also showed that PL turned into an LI effect as the discriminability between the preexposed stimuli increased (see, e.g., Chamizo \& Mackintosh, 1989; Trobalon, Chamizo, \& Mackintosh, 1992; Trobalon, Sansa, Chamizo, \& Mackintosh, 1991). 
In addition to proposing a role of LI in producing the PL effect, McLaren and Mackintosh (2000) proposed two additional contributing mechanisms: unitization (the formation of excitatory links among the single elements that form a stimulus) and inhibitory associations (formed among the unique features of a compound). There is extensive experimental evidence for the combined role of these mechanisms in producing the PL effect (see, e.g., Bennett, Scahill, Griffiths, \& Mackintosh, 1999; Bennett, Wills, Wells, \& Mackintosh, 1994; Dwyer, Bennett, \& Mackintosh, 2001).

However, it is important to note that in several circumstances doubt has been cast on the role of LI in producing PL effects. For example, although a strong PL effect occurs when the discriminative stimuli are alternated in preexposure, no PL effect occurs when the stimuli were preexposed in separate blocks (see, e.g., Honey \& Bateson, 1996; Mondragon \& Hall, 2002; Symonds \& Hall, 1995). Apparently, close comparison of the stimuli in preexposure can be important.

Further complexities in the relation of LI to PL effects in discrimination learning have been reported in experiments in which retention intervals have been inserted into the preexposure/training process. For example, Killcross, Kiernan, Dwyer, and Westbrook (1998), using the context as the CS in a freezing procedure, found that LI was disrupted by a 14-day interval between discrimination training and testing (Experiment 1A), but a similar retention interval in a related experiment (Experiment 1B) did not affect PL magnitude. A similar pattern of results occurred when the retention interval was placed between preexposure and conditioning: LI was again attenuated, but PL was not significantly affected (Experiment 2). However, De la Casa and Alonso (2005), using a taste aversion procedure, found that inserting a 14-day interval between conditioning and testing disrupted both LI and PL.

The purpose of the present research was to further evaluate the hypothesis of McLaren and Mackintosh (2000) that LI plays an important role in producing PL effects. Our first goal was to establish data supporting the hypothesis of an interaction among preexposure, similarity of elements in the stimuli, and subsequent discrimination learning. Specifically, when the preexposed stimuli have many similar elements, subsequent discrimination learning should be facilitated relative to a control group (a PL effect), and when the preexposed stimuli are composed largely of dissimilar elements, subsequent discrimination learning should be retarded relative to a control group (an LI effect). Our second goal was to evaluate further the hypothesis that LI plays an important role in producing PL effects by examining the effect of imposing a 21-day retention interval, either between discrimination training and a final test (Experiment 1) or between preexposure and discrimination training (Experiment 2).

We used an appetitive Y-maze discrimination procedure because previous experiments showed that spatial choice is sensitive to both LI and PL as a function of the difficulty of the discrimination (see Oswalt, 1972). Chamizo and Mackintosh (1989) reported that LI and PL effects after stimulus preexposure in a Y-maze discrimination task varied as a function of the number of elements shared by the discriminative stimuli. In their Experiment 1, the animals were preexposed to two different stimuli (rubber and sandpaper covering the maze's arms), which were later employed in a single discrimination task. As a result of preexposure, the animals performed better in the discrimination stage (a PL effect) in comparison with a control group with similar exposure to the maze but without preexposure to the stimuli. In Experiment 2, Chamizo and Mackintosh made the discrimination easier by painting the walls of the maze black and white, turning the previous PL effect into an LI effect (see also Trobalon et al., 1991).

In the present experiments, we tested both effects using a common preexposure to all stimuli. Both experiments began with preexposure of all the experimental subjects to three maze arms, each with a different stimulus pattern on the walls. Two of the arm patterns shared similar stimulus elements (narrow alternating black and white stripes running either horizontally or vertically), and the third (dissimilar) arm pattern consisted of more widely spaced, large, "splotchy" dots. The control groups were exposed to the same arms with plain white walls. On the basis of the hypothesis of McLaren and Mackintosh (2000) and the results of Chamizo and Mackintosh (1989), we expect to show retarded discrimination learning in the LI group in comparison with the LI-control group, and enhanced learning in the PL group in comparison with the PL-control group.

Our expectations for the effects of imposed retention intervals on PI and/or LI were less clear. The results of Killcross et al. (1998) and De la Casa and Alonso (2005) noted above can be tentatively summarized as follows: LI is more sensitive than PL to disruption by a lengthy retention interval, and neither LI effects nor PL effects appear to depend on whether the retention interval is introduced following preexposure or following training. However, further experiments measuring only the effects on LI of imposing a retention interval suggest additional complexity. LI effects of a retention interval between conditioning and final testing include abolition (see, e.g., Aguado, Symonds, \& Hall, 1994), no effect (see, e.g., Alvarez \& Lopez, 1995), and a super-LI effect (see, e.g., De la Casa \& Lubow, 2000). LI effects of a retention interval between exposure and training also vary. Thus, Aguado et al. (1994, Experiment 3), using a conditioned taste aversion procedure, reported a disruption of LI when a 14-day interval was introduced between preexposure and conditioning stages. Conversely, De la Casa and Lubow (2002, Experiment 3), using a taste aversion procedure, found no effect of a 21-day interval on LI (Experiment 3). Finally, Rosas and Bouton (1997), using an appetitive procedure and a 28-day retention interval in two experiments, found attenuation of LI in one of the experiments, but not in the other.

We assume that specific procedural details, such as the nature of the US, the context, and the length of the retention interval, contributed to the varied effects of retention 
intervals on LI (for a review, see Lubow \& De la Casa, 2005). However, these complexities and qualifications, although interesting in their own right, are not relevant to testing the hypothesis of McLaren and Mackintosh (2000) that PL is directly related to LI. For present purposes, the key prediction from this hypothesis is that LI and PL effects should change in parallel, and certainly not in opposite directions.

\section{EXPERIMENT 1}

In the first experiment, we examined the effects of preexposure (PE) to maze arm patterns versus unpatterned (white) maze arms (nonpreexposure, or NPE). In both the PE and NPE conditions, one group was trained to discriminate the similar horizontal versus vertical stripes (PL-PE and PL-NPE, respectively). The other group in each exposure category was trained to discriminate the dissimilar stimuli of horizontal or vertical stripes versus the splotches (LI-PE and LI-NPE, respectively). Following a retention interval of 21 days, a test of the discrimination was conducted to evaluate the effects LI and PL. A summary of the design is presented in Table 1.

\section{Method}

\section{Subjects}

Thirty-two female Wistar naive adult rats were used in this experiment. At the start of the experiment, their mean ad lib body weight was $262 \mathrm{~g}$ (range, 231-298 g). The animals were then food deprived to $85 \%$ of their weight by providing reduced rations in their home cages once a day at a fixed time. Water was continuously available in the home cages. Each animal was handled for $2 \mathrm{~min}$ on each of the 7 days prior to the beginning of preexposure. Three animals were dropped from the experiment because they failed to move about the maze on test trials. As a consequence, there were 8 rats in Group LI-PE and 7 rats in each of the other groups.

\section{Apparatus}

During discrimination training, we used a modified Y-maze on a platform $75 \mathrm{~cm}$ off the floor, with a start box $17 \mathrm{~cm}$ wide $\times 22 \mathrm{~cm}$ long with a guillotine start door and two choice arms separating from each other at an angle of $90^{\circ}$. A third arm located between the two choice arms was used during preexposure to facilitate exposure of the rats to all three arm conditions. Each arm was $60 \mathrm{~cm}$ long $\times$ $17 \mathrm{~cm}$ wide and had 28-cm-high clear Plexiglas walls along the sides and ends of the arms. As appropriate for the various conditions, plain white paper or white paper with black printed patterns was attached to the outside of the Plexiglas walls of each arm so that it surrounded the arm but could not be contacted by the animal.
Each arm contained a recessed 3.5-cm-diam food cup located $2 \mathrm{~cm}$ from the end, and access to the arm was controlled by independently operated $8.5-\mathrm{cm}$-high guillotine doors made of sheet metal. Because of the presence of the three possible choice arms (although we used only the two outside arms in discrimination training), there was a $45-\mathrm{cm}$-long fan-shaped choice area between the door to the start box (at the narrow end) and the doors to the arms (at the wide end).

The maze was located in the center of a room illuminated by four symmetrically situated ceiling lights. A black curtain completely surrounded the maze to prevent the animals from using extramaze landmarks during the discrimination stage. During the discrimination training stage, the maze was used as a Y-maze, allowing the subjects to choose between two arms, one of which was consistently rewarded and the other extinguished. Two 97-mg Noyes pellets (Formula P) were used to designate the correct arm.

During the preexposure stage for the rats in the PE group, the walls and far end of each choice arm were completely covered by one of three visual patterns printed in black on white paper protected by transparent plastic (Kittrich, Los Angeles). One pattern was composed of $18-\mathrm{cm}$ horizontal lines alternating between black and white, the second pattern consisted of the same lines in a vertical orientation, and the third was composed of $2.5-\mathrm{cm}$-diam circles, each $6.3 \mathrm{~cm}$ (center to center) from the circles to its left and right and $3.3 \mathrm{~cm}$ (center to center) from the circles above and below it. Figure 1 displays pictures of the visual patterns used in the experiment. For the rats in the NPE groups, during the preexposure stage the walls of each arm were covered with plain white paper protected by the transparent plastic. In the discrimination stage, the two stimuli having more common elements (A and B in Figure 1) were used in the difficult discrimination (for the PL groups), whereas two stimuli sharing few elements (A and C, or B and C) were used in the easy discrimination (for the LI groups).

\section{Procedure}

Preexposure. Preexposure lasted for 6 days, with two sessions run each day. The first daily session was run at about 10:00 a.m. and the second at about 3:00 p.m. During each session, the animals in all the groups were placed singly in each one of the arms with the door shut for $2 \mathrm{~min}$. Therefore, each animal was preexposed for a total of 24 min to each of the arms. For the PE animals, each arm contained one of the stimulus patterns. The order of exposure to the arms and the location of the different stimuli were fully counterbalanced across sessions (morning vs. afternoon) and days. The animals in the NPE condition were exposed in the same way as the PE animals, but with plain white walls in all arms.

Discrimination training. Discrimination training involved the use of the two outside arms and lasted for 10 days, with eight trials on each day for each rat. For each trial, the rat was placed in the start box, facing the arms. One of the visual patterns covering the arms was consistently rewarded, and the other was consistently not rewarded. Once the animal made a choice, the chosen arm was closed and the rat remained undisturbed for the shorter of $20 \mathrm{sec}$ or

Table 1

Design of Experiment 1

\begin{tabular}{lcccc}
\hline Group & Preexposure & $\begin{array}{c}\text { Discrimination } \\
\text { Training }\end{array}$ & $\begin{array}{c}\text { Delay } \\
\text { (Days) }\end{array}$ & Retest \\
\hline LI-PE & A, B, C & A vs. C or B vs. C & 21 & A vs. C or B vs. C \\
LI-NPE & - & A vs. C or B vs. C & 21 & A vs. C or B vs. C \\
PL-PE & A, B, C & A vs. B & 21 & A vs. B \\
PL-NPE & - & A vs. B & 21 & A vs. B \\
\hline
\end{tabular}

Note-LI, latent inhibition; PL, perceptual learning; PE, preexposed; NPE, nonpreexposed; A, pattern composed of vertical lines; B, pattern composed of horizontal lines; C, pattern composed of circles. 


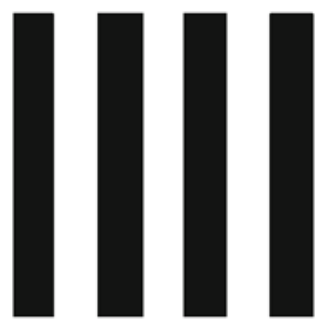

A

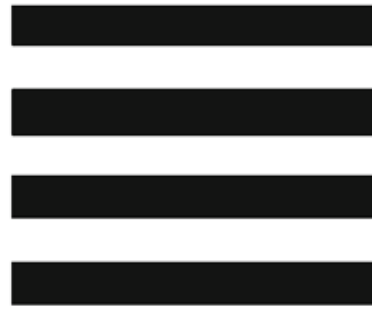

B

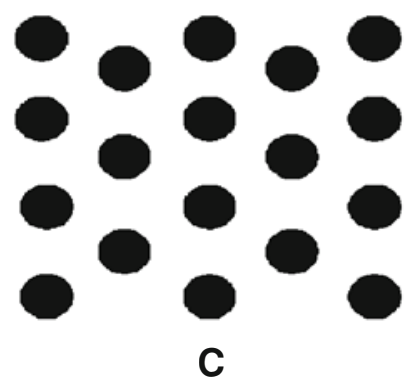

Figure 1. Visual patterns used in Experiments 1 and 2. All the animals in the preexposed groups were exposed to the three patterns before discrimination training. The comparison of $A$ versus $B$ was used for the discrimination in the PL condition (difficult discrimination). Comparisons of $A$ versus $C$ and $B$ versus $C$ were used for discrimination training in the LI condition (easy discriminations). See text for details.

the time necessary to eat the pellets. The rat was then removed and placed again at the start point to begin the next trial.

For those animals in the LI-PE and LI-NPE groups, the discrimination training involved easy discriminations between $\mathrm{A}$ and $\mathrm{C}$ stimuli for half of the subjects and between B and C stimuli for the other half. The location of the stimuli as well as the reinforced pattern were fully counterbalanced across subjects. For those animals in the PL-PE and PL-NPE groups, the discrimination involved A versus B stimuli. Again, location and reinforced pattern were completely counterbalanced. The mean percent of correct trials on each session was computed as an index of learning.

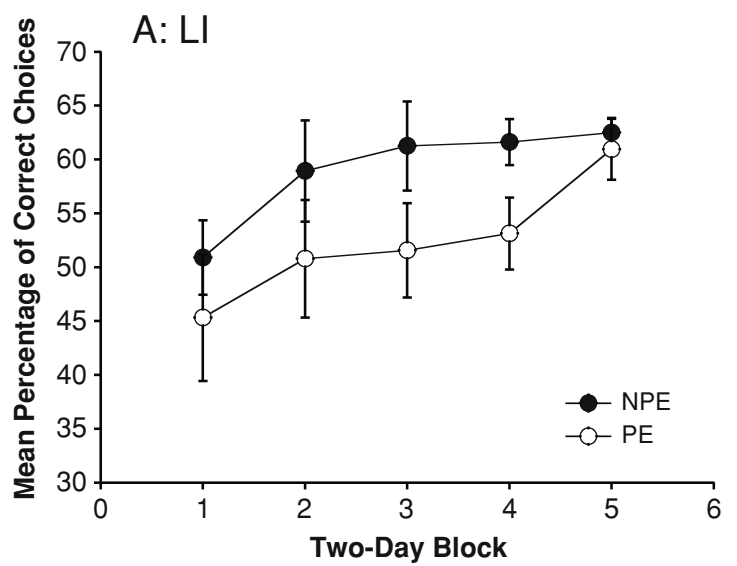

Retest. After the last discrimination session, the animals remained undisturbed in their home cages for the next 21 days. Food access was unrestricted during the first 14 days. On Day 15 of the retention interval, the food deprivation schedule was newly implemented in such a way that, on Day 21, all the animals were at the same deprivation level ( $85 \%$ of ad lib weight) and remained so for the remaining experimental stages. On Day 22, each animal was retested on its discrimination (eight trials for each rat).

\section{Results and Discussion}

Figure 2 shows the mean percent of correct choices over 2-day blocks during the discrimination training for each group. As can be seen in the figure (panel A), for the LI animals there was a progressive increase in correct choices across trials. However, learning proceeded faster for Control Group LI-NPE than for Group LI-PE, revealing the expected retardation in learning when the stimuli involved in the discrimination were clearly different (the easy discrimination). Conversely, as can be seen in panel B, learning for Group PL-NPE was slower than that for Group PL-PE-evidence of the PL effect expected when the stimuli in discrimination training have many common elements (the difficult discrimination).

These conclusions were supported by a 10 (trials) $\times 2$ (preexposure: PE vs. NPE) $\times 2$ (discrimination type: easy vs. difficult) mixed ANOVA. The within-subjects analysis revealed a significant main effect of trials $[F(9,225)=$ 2.77, $p<.01]$. None of the interactions involving trials was significant [all $\left.F_{\mathrm{s}}(9,225)<1\right]$. The between-subjects analysis revealed a significant preexposure $\times$ discrimination interaction $[F(1,25)=20.17, p<.001]$. The interaction revealed that preexposure to the stimuli resulted in faster learning when the animals were confronted with the difficult discrimination (PL effect) but retarded learning when the animals learned the easier discrimination (LI effect). Pairwise comparisons among group means using Duncan's test revealed significant differences between the LI-PE and LI-NPE groups $(p<.05)$ and between the PL-PE and PL-NPE groups $(p<.01)$, showing LI and PL effects, respectively. An additional comparison

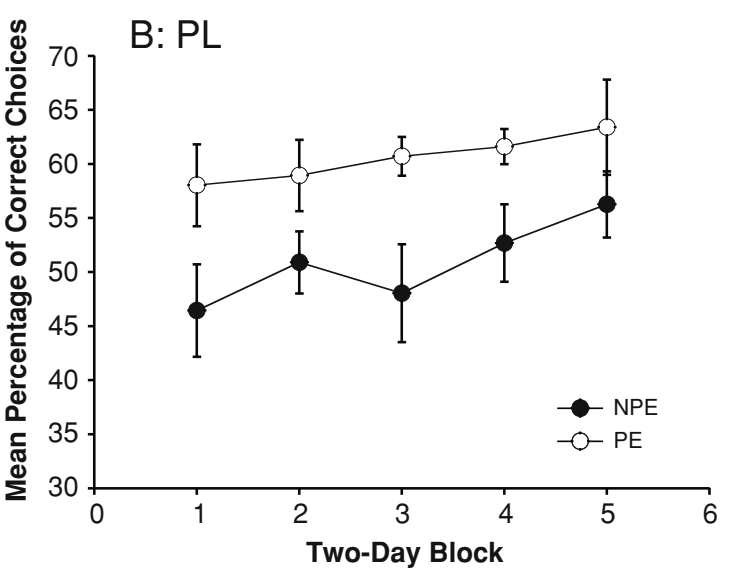

Figure 2. Experiment 1: mean percentages of correct choices in the discrimination stage for (A) LI groups and (B) PL groups over 2-day blocks. Error bars represent standard errors of the mean. 
between the NPE groups showed a significant difference $(p<.01)$ due to lower learning in the PL-NPE than in the LI-NPE group. This difference indicates that, as was expected, the discrimination between those stimuli sharing many elements (A vs. B in Figure 1) was more difficult than the discrimination between those stimuli that shared few elements (A vs. C and B vs. C, shown as the LI groups in Figure 1).

Twenty-one days after the last day of the discrimination stage, a 1-day retest was conducted following the same procedure as on the last day of the discrimination stage. Figure 3 shows the mean percent of correct choices as a function of the preexposure condition on the last day of the discrimination stage and on the retest day (collapsed across trials). Panel A corresponds to the LI groups, and panel B to the PL groups. As can be seen in the figure, the retention interval following training did not affect the animals' responses in any condition. These impressions were confirmed by a 2 (last discrimination day vs. retest, within subjects) $\times 2$ (preexposure) $\times 2$ (discrimination type) mixed ANOVA, revealing a significant preexposure $X$ discrimination interaction $[F(1,25)=5.23, p<.05]$. The interaction was due to the different effect of preexposure as a function of the stimuli involved in the discrimination (i.e., the LI effect and the PL effect). No other main effects or interactions were significant (all $p s>.17$ ).

\section{EXPERIMENT 2}

Experiment 1 showed that discrimination learning between two stimuli after preexposure to both can be retarded (LI groups) or enhanced (PL groups) as a function of the proportion of elements shared by the stimuli to be discriminated. Specifically, when the stimuli shared many elements (i.e., during a difficult discrimination), PL was found, whereas when they shared few elements (i.e., during an easy discrimination) LI was found. In addition, a 21-day interval interpolated between the end of discrimination training and the retest did not change the discrimination ability of either the LI or the PL group.

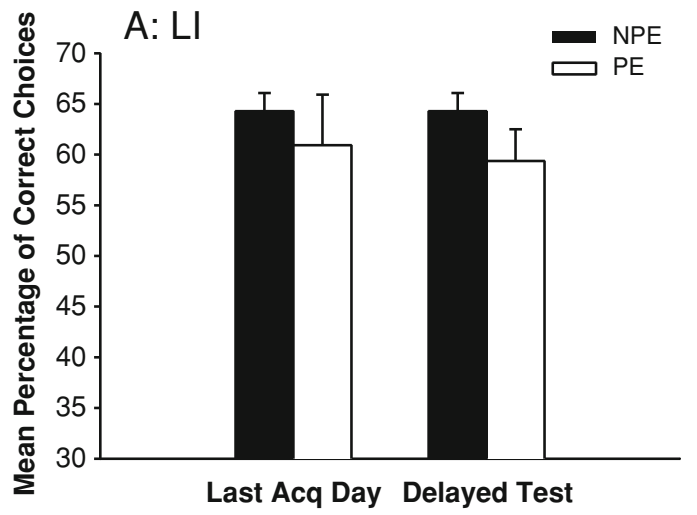

It might be argued that the procedure employed in Experiment 1 was not the best choice for evaluating the impact of a retention interval between conditioning and test stages in the maze discrimination paradigm. All previous research conducted to analyze the effects of a retention interval between conditioning and test stages employed one-trial aversive conditioning in a three-stage procedure (preexposure, conditioning, and test). This procedure allows differentiation of associations established during the preexposure and conditioning stages that might be competing for expression at testing (see, e.g., De la Casa, Diaz, \& Lubow, 2005). In contrast, our procedure included only two stages (preexposure and a lengthy conditioning/test phase). Our procedure likely produced asymptotic levels of conditioning for all groups prior to the retention interval, thus favoring similar degrees of expression and little forgetting during the retention interval (for a discussion of additional differences between the two- and three-stage LI procedures, see Lubow, 1997; Lubow \& Kaplan, 2005).

An alternative strategy is to evaluate the influence of a retention interval introduced after preexposure on subsequent discrimination training effects (LI and PL). To this end, we introduced the 21-day delay between preexposure and discrimination training. To the extent that preexposure memory for similar versus dissimilar stimuli is differentially lost, we should find different effects of the retention interval on the acquisition of LI and PL effects.

Previous research has shown that a retention interval introduced between preexposure and conditioning stages can disrupt LI (e.g., Aguado et al., 1994, Experiment 3; Rosas \& Bouton, 1997, Experiment 2; for demonstrations with humans, see Escobar, Arcediano, \& Miller, 2002; Pineño, De la Casa, Lubow, \& Miller, 2006, Experiment 2). As far as we know, there is no evidence of the effect of such a manipulation on PL, but we expect that if the retention interval is effective in attenuating LI, a similar disruptive effect may be obtained in the corresponding PL group due to the hypothesized crucial contribution of LI to PL (see McLaren \& Mackintosh, 2000).

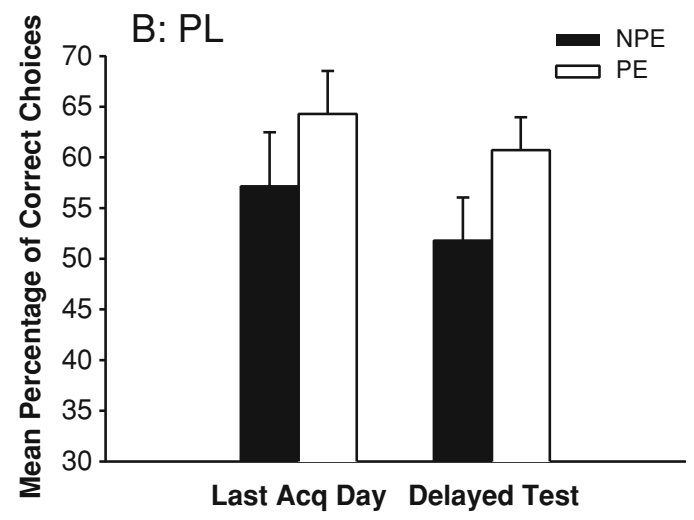

Figure 3. Mean percentages of correct choices (collapsed across trials) for the last day of the acquisition stage and for the retest conducted after a 21-day delay. (A) Data from the LI groups. (B) Data from the PL groups. Error bars represent standard errors of the mean. 
To evaluate these predictions, the present experiment included four groups, all preexposed to the visual patterns that subsequently served in the discrimination task. For half of the subjects, the discrimination training stage was conducted 1 day after preexposure (1-day condition, as in Experiment 1), and for the other half it was conducted 21 days after preexposure (21-day condition). To reproduce the LI and PL preexposures in Experiment 1, half of the rats in each delay condition were trained to discriminate between the stimuli having many common elements (PL groups), and the other half between the stimuli having fewer common elements (LI groups), in the same way as was described for Experiment 1. Table 2 summarizes the design of Experiment 2.

We could not include the NPE groups (preexposed only to the white, unpatterned maze arms) in Experiment 2 because of time constraints. Therefore, we could not directly measure LI and PL effects. However, given the clear results of Experiment 1, which showed that our procedure was effective in inducing both LI and PL effects, and the use of the same (1-day) procedure of no retention interval for half of the rats in the present experiment, we can test the hypothesis that insertion of a retention interval will have parallel effects on the easy (LI) and the difficult (PL) discriminations.

\section{Method}

\section{Subjects}

Thirty-two naive adult female Wistar rats were used in this experiment. Their mean body weight was $258 \mathrm{~g}$ (range, 225-307 g). The animals were deprived, handled, and maintained as in Experiment 1. Three animals that did not make any choice during the discrimination stage were removed from the experiment. Thus, each of three groups consisted of 7 rats, whereas one group (LI/1 day) consisted of 8 rats.

\section{Apparatus and Procedure}

The same maze, stimuli, and preexposure procedure described for the LI-PE and PL-PE animals in Experiment 1 were used in Experiment 2. For the LI and PL groups in the 21-day condition, the preexposure stage started on Day 1 and lasted until Day 6, to be followed by 21 retention days. For the LI and PL groups in the 1-day condition, the preexposure stage took place from Day 21 to Day 26. The discrimination stage started for all the animals on Day 27 and lasted for 10 days, with eight discrimination trials taking place each day. As in Experiment 1, the discrimination stimuli for the PL groups were horizontal versus vertical stripes (A vs. B in Figure 1). The discrimination stimuli for the LI groups were a striped pattern versus the dotted pattern (A vs. C or B vs. C in Figure 1). The discrimination stage was conducted as described for Experiment 1.

Table 2

Design of Experiment 2

\begin{tabular}{lccc}
\hline \multicolumn{1}{c}{ Group } & Preexposure & $\begin{array}{c}\text { Delay } \\
\text { (Days) }\end{array}$ & $\begin{array}{c}\text { Discrimination } \\
\text { Training }\end{array}$ \\
\hline LI/1 day & A, B, C & 1 & A vs. C or B vs. C \\
LI/21 days & A, B, C & 21 & A vs. C or B vs. C \\
PL/1 day & A, B, C & 1 & A vs. B \\
PL/21 days & A, B, C & 21 & A vs. B \\
\hline
\end{tabular}

Note-LI, latent inhibition; PL, perceptual learning; A, pattern composed of vertical lines; B, pattern composed of horizontal lines; C, pattern composed of circles.

\section{Results and Discussion}

Figure 4 shows the mean percent of correct choices over 2-day blocks during the discrimination training for the LI (panel A) and PL (panel B) groups. As can be seen in the figure, the expected differences between the LI and PL groups were observed when testing was conducted 1 day after preexposure (i.e., Group LI/1 day vs. Group PL/1 day), as was the case for Experiment 1. However, the 21-day delay introduced between preexposure and discrimination for Groups LI/21 days and PL/21 days appeared to reduce the LI performance observed in the LI/1-day group, but not the PL performance observed in the PL/1-day group.

These impressions were confirmed by statistical analyses. A 10 (trials) $\times 2$ (discrimination type: PL vs. LI) $\times$ 2 (delay: 1 vs. 21 days) mixed ANOVA was conducted on the means of correct choices. The within-subjects analysis revealed a significant main effect of trials $[F(9,225)=$ $10.14, p<.001]$ due to the progressive improvement of the discrimination across trials. None of the interactions involving trials was significant [all $\left.F_{\mathrm{S}}(9,225)<1\right]$. The between-subjects analyses revealed a main effect of discrimination type $[F(1,25)=4.65, p<.05]$ due to the faster learning shown by the PL groups $(M=60.22 \%$ correct choices, $S D=4.73$ ) in comparison with that of the LI groups $(M=56.35 \%$ correct choices, $S D=5.98)$. The main effect of delay was nonsignificant $[F(1,25)<1]$, but the two-way delay $\times$ discrimination type interaction was significant $[F(1,25)=13.02, p<.01]$.

Pairwise comparisons among the group means using Duncan's test $(p<.05)$ were performed to identify the source of the delay $\times$ discrimination type interaction. The analysis revealed significant differences between the LI/1-day and PL/1-day groups on the one hand, and the LI/1-day and LI/21-day groups on the other. However, the difference between the PL/1-day and PL/21-day groups was not significant. In short, the LI effect of retarding discrimination learning was markedly attenuated by a 21-day delay between preexposure and discrimination training, but the PL effect of facilitating learning was not affected by the delay.

\section{GENERAL DISCUSSION}

The subjects in Experiment 1 showed a PL effect (facilitation of discrimination learning relative to a control group) when preexposed to stimuli sharing many elements. They also showed an LI effect (retardation of discrimination learning relative to a control group) when preexposed to stimuli with largely disparate elements. According to McLaren and Mackintosh (2000), three associative processes can contribute to a PL effect: (1) the formation of inhibitory associations between the unique elements of the stimuli, (2) the formation of separate stimulus representations for each stimulus as a result of a unitization process, and (3) a strong reduction of the associability of common elements of preexposed stimuli (LI) relative to that of the unique elements, because of the double amount of preexposure to the common features. 

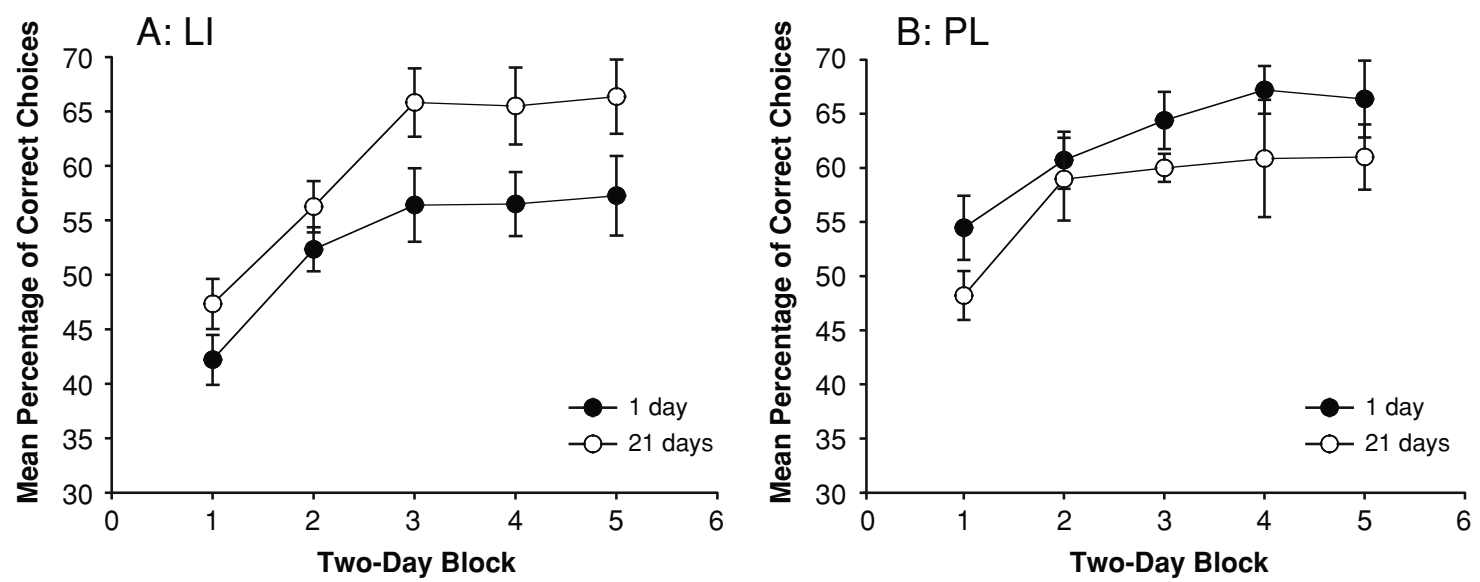

Figure 4. Experiment 2: mean percentages of correct choices in the discrimination stage for (A) LI groups and (B) PL groups over 2-day blocks. Error bars represent standard errors of the mean.

Our findings of the occurrence of PL and LI as a function of type of discrimination confirms previous reports in mazes (e.g., Chamizo \& Mackintosh, 1989) and appears best explained by the third mechanism hypothesized by McLaren and Mackintosh (2000) — namely, that a double preexposure to elements shared by stimuli differentially guides attention to the unique elements that distinguish them. It may be worth noting that predictions based on this hypothesis could profit from further converging analyses of the perception and discriminability of different stimulus elements (including spatial, temporal, modality, and quality aspects) as well as from the effects of different patterns of exposure. For example, in the present case it is possible that horizontal and vertical black and white stripes have a unique blend of multiple common elements and clear discriminability for the rat visual system.

In addition to confirming the relation of preexposure to stimuli with different proportions of shared elements to LI and PL effects in spatial discrimination, in our experiments we attempted to evaluate the role of LI in the PL effect by inserting a 21-day retention interval following discrimination training in Experiment 1 and following preexposure in Experiment 2. In Experiment 1, we assessed the effects of a posttraining retention interval by comparing the effects of preexposure on LI and PL at the end of discrimination training and after the 21-day retention interval. In Experiment 2, the effects on LI and PL of a 21-day retention interval between preexposure and discrimination training were compared with those of a 1-day retention interval.

Previous work in a number of discrimination paradigms has shown a variety of effects of a retention interval on LI and PL phenomena. The results could be summarized approximately as follows: Inserting a retention interval in experiments in which both PL and LI groups are trained following appropriate preexposure to stimuli can interfere with both LI and PL in parallel, or can interfere with LI without affecting PL. In most experiments, the insertion or placements of retention intervals appeared to have little effect. The data from both our experiments are compatible with this generalization. In Experiment 1, imposing a 21-day retention interval between discrimination training and final testing affected neither LI nor PL. However, in Experiment 2 imposing the same 21-day retention interval between preexposure and discrimination training significantly reduced subsequent performance on the LI (dissimilar stimuli) discrimination, but not that on the PL (similar stimuli) discrimination.

There is more work to be done in this area, since experiments testing the effects of retention interval show a more complex pattern of results than do experiments examining only LI effects (for a review, see Lubow \& De la Casa, 2005). A general factor that apparently modulates LI effects regardless of where the retention interval is placed is the context in which subjects spend the retention interval. When the interval context is the same as that of the remaining experimental situations, it results in attenuation of LI (see, e.g., Aguado et al., 1994). However, when the retention context is different from the context for preexposure, conditioning, and testing, imposing a retention interval enhances LI (see, e.g., De la Casa \& Lubow, 2000).

Other differences may be related to the nature of the system related to the US. In the case of a retention interval between conditioning and testing, De la Casa et al. (2005) noted that LI varies as a function of the motivational features of the US involved in the target association. When LI is studied in an aversive setting, delayed testing could favor a fear incubation process that could differentially affect the preexposed and nonpreexposed groups, resulting in an apparent reduction of LI. Notably, to our knowledge there is no evidence of LI attenuation when an appetitive US is involved in learning and the delay is inserted between conditioning and testing stages. The null result in our Experiment 1 is consistent with this view.

In aversive conditioning, the difference in the effects of a retention interval between the preexposure and conditioning phases may occur because there are no grounds here for the action of a fear incubation effect: The US has 
not been presented before the retention interval. Moreover, attenuation of LI when a retention interval is interpolated between preexposure and acquisition is consistent with Bouton's (1993) retrieval failure model, which considers time as a part of the context. If the representation of context changes as a function of the passage of time, the introduction of the delay following preexposure can be considered as a context change between preexposure and conditioning, a condition that promotes LI attenuation (see, e.g., Channell \& Hall, 1983). From this viewpoint, attenuation of LI in the long delay condition observed in Experiment 2 was the result of a change of context induced by the retention interval at the time of acquisition. Our observation that PL was not affected by the time-induced context change is consistent with previous evidence showing that the PL effect survived a long delay between preexposure and conditioning (Killcross et al., 1998, Experiment 2). It may also be consistent with some data showing that a context change between exposure and acquisition is a condition that promotes PL (Lubow, Rifkin, \& Alek, 1976).

A possible way to integrate the results of Experiment 2 with McLaren and Mackintosh's (2000) view of PL comes from the proposal of Trobalon et al. (1992) that a context change could produce both a disruption and an enhancement of the PL effect as a function of total preexposure time. To the extent that perceptual learning depends on a differential loss of associability by common and unique features, it will be reduced by extended exposure to the discriminative stimuli. A moderate amount of preexposure will have a greater effect on the associability of the common elements than on that of the unique elements, thus attenuating the perceptual learning effect. But with extended preexposure, a change of context will increase the difference between unique and common elements, consequently enhancing the perceptual learning effect.

This possibility, however, is difficult to apply to the present data because the amount of exposure in our experiments was $24 \mathrm{~min}$ to each of the three patterns (for a total of $72 \mathrm{~min}$ ). This can be considered a moderate amount of exposure in comparison with the $50 \mathrm{~h}$ of exposure in Channell and Hall's (1983) experiments, which revealed a strong PL effect when preexposure was conducted in a context different from that of discrimination training (home cages and test apparatus, respectively) and abolition of PL when preexposure and discrimination training were both conducted in the test enclosure. In fact, Trobalon et al. (1992) explicitly considered that $64 \mathrm{~min}$ is a small amount of exposure that should reduce the PL effect after the context change.

From this viewpoint, the survival of PL after the 21-day retention interval following preexposure does not support the view that LI is a major mechanism producing the facilitation of discrimination after preexposure. It also does not support the idea that the actions of unspecified nonassociative mechanisms promote a gradual increase in the differentiation of those stimulus features most relevant to stimulus discrimination (see Hall, 2003; Saksida, 1999). Specifically, Saksida considered that stimulus pre- exposure can lead to either facilitation or retardation of learning by means of a nonassociative mechanism that promotes stimulus differentiation. When the preexposed stimuli are similar, each presentation will generate (1) a reduction in stimulus associability and (2) different stimulus representations that will facilitate subsequent discrimination. Conversely, when the preexposed stimuli are very different, successive stimulus exposures will produce only a reduction in their associability.

In contrast, Hall (2003) considers that a process of stimulus differentiation (Gibson, 1969) is mainly responsible for the PL effect. During preexposure, this process will produce an increase in the perceptual effectiveness of the unique elements of the preexposed stimuli and a parallel weakening in the effectiveness of the common features. The two proposed mechanisms that explain the changes in perceptual effectiveness are the following: First, a habituation process would be responsible for the decline in effectiveness of the common elements; and, second, the increase in effectiveness of the unique elements would come from a negative habituation process produced by the indirect activation of the unique elements of the absent stimulus, which in turn is induced by the presentations of the second stimulus (Blair \& Hall, 2003; Hall, 2003).

Some experimental results have given support to the explanations of the PL effect from nonassociative views. For instance, PL has been more readily obtained as a function of the order of preexposure to the stimuli; thus, the PL effect is stronger when stimuli are presented in a way that promotes comparison between them than when preexposure does not favor such comparison (see, e.g., Symonds \& Hall, 1995). More specifically, Mondragon and Hall (2002) presented two flavor compounds (AX and $\mathrm{BX})$ in either an intermixed (AX, BX, AX, BX, ...) or a blocked ( $\mathrm{AX}, \mathrm{AX}, \ldots, \mathrm{BX}, \mathrm{BX}, \ldots$ ) fashion and found that PL effect was stronger in the intermixed than in the blocked condition. If LI is a crucial factor in PL, no difference should be observed as a function of the preexposure sequence, since the total amount of preexposure to the stimuli is the same in both conditions.

McLaren and Mackintosh (2000) invoked additional effects to account for the effects of sequential versus intermixed preexposure. They argued that intermixing stimuli promoted mutually inhibitory associations between their unique elements. Similarly, Mondragon and Hall (2002), making use of the notions of perceptual learning (Gibson, 1969), argued that intermixed preexposure promoted comparison of stimuli, thereby increasing differentiation between them. However, such explanations do not account for the finding that LI, but not PL, is attenuated by a delay between preexposure and discrimination training.

In summary, our data do not give strong direct support to the hypothesis that LI is a major underlying mechanism of PL, because PL occurred without LI. However, our results are not conclusive, because a procedure that reduces LI does not necessarily abolish its potential effects. However, on an empirical level, it is clear that introducing a 21-day retention interval between preexposure and discrimination training disrupts LI without disrupting 
PL. Nevertheless, when the retention interval occurs after discrimination, neither LI nor PL effects are disrupted-a finding that might be explained by appealing to the stability of perceptual learning in discrimination or by treating a delay before discrimination (but not a delay after discrimination) as a context change (Bouton, 1993).

\section{REFERENCES}

Aguado, L., Symonds, M., \& Hall, G. (1994). Interval between preexposure and test determines the magnitude of latent inhibition: Implications for an interference account. Animal Learning \& Behavior, 22, 188-194.

Alvarez, R., \& Lopez, M. (1995). Effects of elements or compound preexposure on conditioned taste aversion as a function of retention interval. Animal Learning \& Behavior, 23, 391-399.

Bennett, C. H., Scahill, V. L., Griffiths, D. P., \& Mackintosh, N. J. (1999). The role of inhibitory associations in perceptual learning. Animal Learning \& Behavior, 27, 333-345.

Bennett, C. H., Wills, S. J., Wells, J. O., \& Mackintosh, N. J. (1994). Reduced generalization following preexposure: Latent inhibition of common elements or a difference in familiarity? Journal of Experimental Psychology: Animal Behavior Processes, 20, 232-239.

Blair, C. A. J., \& Hall, G. (2003). Perceptual learning in flavor aversion: Evidence for learned changes in stimulus effectiveness. Journal of Experimental Psychology: Animal Behavior Processes, 29, 39-48.

Bouton, M. E. (1993). Context, time, and memory retrieval in the interference paradigms of Pavlovian learning. Psychological Bulletin, 114, 80-99.

Chamizo, V. D., \& Mackintosh, N. J. (1989). Latent learning and latent inhibition in maze discriminations. Quarterly Journal of Experimental Psychology, 41B, 21-31.

Channell, S., \& Hall, G. (1983). Contextual effects in latent inhibition with an appetitive conditioning procedure. Animal Learning \& Behavior, 11, 67-74.

De la CASA, L. G., \& Alonso, G. (2005). Effects of retention interval on latent inhibition and perceptual learning in the conditioned taste aversion paradigm. Manuscript submitted for publication.

De la Casa, L. G., Diaz, E., \& Lubow, R. E. (2005). Latent inhibition with a conditioned emotional response procedure: The effects of delayed testing. Learning \& Motivation, 36, 60-76.

De La Casa, L. G., \& Lubow, R. E. (2000). Super-latent inhibition with delayed conditioned taste aversion testing. Animal Learning \& Behavior, 28, 389-399.

De la Casa, L. G., \& Lubow, R. E. (2002). An empirical analysis of the super-latent inhibition effect. Animal Learning \& Behavior, 30 112-120.

Dwyer, D. M., Bennett, C. H., \& Mackintosh, N. J. (2001). Evidence for inhibitory associations between the unique elements of two compound flavours. Quarterly Journal of Experimental Psychology, 54B, 97-107.

Escobar, M., Arcediano, F., \& Miller, R. R. (2002). Latent inhibition and contextual associations. Journal of Experimental Psychology: Animal Behavior Processes, 28, 123-136.

Gibson, E. J. (1969). Principles of perceptual learning and development. New York: Appleton-Century-Crofts.

Hall, G. (1991). Perceptual and associative learning. Oxford: Oxford University Press, Clarendon Press.

HALL, G. (2003). Learned changes in the sensitivity of stimulus representations: Associative and nonassociative mechanisms. Quarterly Journal of Experimental Psychology, 56B, 43-55.

Honey, R. C., \& Bateson, P. (1996). Stimulus comparison and perceptual learning: Further evidence and evaluation from an imprint- ing procedure. Quarterly Journal of Experimental Psychology, 49B, 259-269.

Killcross, A. S., Kiernan, M. J., Dwyer, D., \& Westbrook, R. F. (1998). Effects of retention interval on latent inhibition and perceptual learning. Quarterly Journal of Experimental Psychology, 51B, 59-74.

Lubow, R. E. (1989). Latent inhibition and conditioned attention theory. New York: Cambridge University Press.

LuBow, R. E. (1997). Latent inhibition as a measure of learned inattention: Some problems and solutions. Behavioural Brain Research, 88, 75-83.

Lubow, R. E., \& De La CASA, L. G. (2005). There is a time and a place for everything: Bidirectional modulations of latent inhibition by timeinduced context differentiation. Psychonomic Bulletin \& Review, 12, 806-821.

Lubow, R. E., \& GewiRTZ, J. (1995). Latent inhibition in humans: Data, theory, and implications for schizophrenia. Psychological Bulletin, 117, 87-103.

Lubow, R. E., \& KaPlan, O. (2005). The visual search analog of latent inhibition: Implications for theories of irrelevant stimulus processing in normal and schizophrenic groups. Psychonomic Bulletin \& Review, 12, 224-243.

Lubow, R. E., Rifkin, B., \& Alek, M. (1976). The context effect: The relationship between stimulus preexposure and environmental preexposure determines subsequent learning. Journal of Experimental Psychology: Animal Behavior Processes, 2, 38-47.

McLaren, I. P. L., KaYe, H., \& Mackintosh, N. J. (1989). An associative theory of the representation of stimuli: Applications to perceptual learning and latent inhibition. In R. G. M. Morris (Ed.), Parallel distributed processing: Implications for psychology and neurobiology (pp. 102-130). Oxford: Oxford University Press, Clarendon Press.

McLaren, I. P. L., \& Mackintosh, N. J. (2000). An elemental model of associative learning: I. Latent inhibition and perceptual learning. Animal Learning \& Behavior, 28, 211-246.

Mondragon, E., \& Hall, G. (2002). Analysis of the perceptual learning effect in flavour aversion learning: Evidence for stimulus differentiation. Quarterly Journal of Experimental Psychology, 55B, 153-169.

OswaLt, R. M. (1972). Relationship between level of visual pattern difficulty during rearing and subsequent discrimination in rats. Journal of Comparative \& Physiological Psychology, 81, 122-125.

Pineño, O., De la Casa, L. G., Lubow, R. E., \& Miller, R. R. (2006). Some determinants of latent inhibition in human predictive learning. Learning \& Motivation, 37, 42-65.

Rosas, J. M., \& Bouton, M. E. (1997). Additivity of the effects of retention interval and context change on latent inhibition: Toward resolution of the context forgetting paradox. Journal of Experimental Psychology: Animal Behavior Processes, 23, 283-294.

SAKSIDA, L. M. (1999). Effects of similarity and experience on discrimination learning: A nonassociative connectionist model of perceptual learning. Journal of Experimental Psychology: Animal Behavior Processes, 25, 308-323.

SchmaJuK, N. A. (2002). Latent inhibition and its neural substrates. Boston: Kluwer.

Symonds, M., \& Hall, G. (1995). Perceptual learning in flavor aversion conditioning: Roles of stimulus comparison and latent inhibition of common stimulus elements. Learning \& Motivation, 26, 203-219.

Trobalon, J. B., Chamizo, V. D., \& Mackintosh, N. J. (1992). Role of context in perceptual learning in maze discriminations. Quarterly Journal of Experimental Psychology, 44B, 57-73.

Trobalon, J. B., Sansa, J., Chamizo, V. D., \& Mackintosh, N. J. (1991). Perceptual learning in maze discriminations. Quarterly Journal of Experimental Psychology, 43B, 389-402.

(Manuscript received March 28, 2005; revision accepted for publication September 12, 2005.) 